Supplementary material for Yang Y. et al. Six SQSTM1 mutations in a Chinese amyotrophic lateral sclerosis cohort, Amyotrophic Lateral Sclerosis and Frontotemporal Degeneration, 2015, doi: 10.3109/21678421. 2015.1009466

\section{Six SQSTM1 mutations in a Chinese amyotrophic lateral sclerosis cohort}

Patient 1 was an E81K carrier of Han ethnicity. At age 58 years, she first manifested with weakness of the right arm, and then she reported weakness in the remaining limbs and slurred speech six months later. She presented at our outpatient clinic seven months from symptom onset. She had no family history of neuromuscular disease or Paget's disease of bone (PDB). Neurologic examination revealed weakness and amyotrophy in all four limbs and mild tongue atrophy with increased deep tendon reflexes in four limbs, occasional spasticity and bulbar symptoms. She also showed increased palmomental, sucking, and jaw jerk reflexes and diminished abdominal reflexes. The Rossolimo, Hoffmann, Babinski and Chaddock signs were positive. Forced vital capacity was $75 \%$. The sensory, autonomic, and extrapyramidal systems were normal. Electromyography (EMG) revealed diffuse neurogenic changes in the right first interosseous muscle, left anterior tibia and right rectus abdominis. Motor nerve conduction velocity (MCV) showed decreased waves of compound muscle action potentials (CMAP) in the right ulnar nerve. The results of the plain whole-body X-ray did not show pathologic bone formation, and blood chemical analysis showed that alkaline phosphatase levels were normal. Blood tests excluded tumor, thyroid dysfunction and immune system disease. This patient was diagnosed with clinically definitive ALS. Currently, the patient has difficulty swallowing and exhibits decreased enunciation.

Patient 2 was a 43 -years-old Han female with a $\mathrm{N} 239 \mathrm{~K}$ substitution. She could not lift her right arm and had right leg weakness. Additionally, she had weakness and amyotrophy in the left limbs after six months. She presented at our outpatient clinic 22 months from symptom onset. She had no family history of neuromuscular disease or PDB. The symptoms progressed gradually with respiratory weakness. Neurologic examination revealed weakness and amyotrophy in all four limbs, with occasional spasticity. Patient 2 showed increased deep tendon reflexes in all four limbs with diminished abdominal reflexes. Palmomental, sucking and plantar reflexes were normal. At the time of examination, no bulbar symptoms were observed. Forced vital capacity was $57 \%$. The sensory, autonomic, and extrapyramidal systems were normal. EMG showed diffuse neurogenic changes in the right anterior tibia and rectus abdominis, and the three parts of the motor unit potential (MUP) were abnormal. The whole-body X-ray and alkaline phosphatase levels did not show signs of PDB. Blood tests excluded tumor, thyroid dysfunction and immune system disease. This patient was diagnosed as clinically probable ALS.

Patient 3 was a 60 -years-old male of Han ethnicity carrying a G297S substitution. Upon initial examination, this patient could not lift his right arm and presented weakness in the legs. He showed limitations in the right arm and left leg, reflecting postpoliomyelitis syndrome. This individual had no family history of neuromuscular disease or PDB. Neurological examination showed amyotrophy in all four limbs, diminished deep tendon reflexes and normal plantar reflexes. Muscle strength of the right arm and legs was IV. These symptoms progressed over the next six months, and neurologic examination showed increased popliteal and biceps reflexes, with bilateral positive Babinski and Chaddock signs. Muscle strength of the right arm and legs was $\mathrm{IV}^{-}$. He had no bulbar symptoms or respiration weakness, and the sensory, autonomic, and extrapyramidal systems were normal. Forced vital capacity was $96 \%$. EMG showed neurogenic changes in both anterior tibia, both gastrocnemius and right biceps brachii. MCV showed decreased CAMP waves in the left median nerve. The whole-body X-ray and alkaline phosphatase levels did not show signs of PDB. Blood tests were normal; thus, this patient was diagnosed with probable ALS.

Patient 4 was a 40 -years-old female E372D carrier of Han ethnicity. She initially had weakness of the right arm and then weakness in the remaining limbs six months later. Bulbar signs and respiration problems occurred six months before she died. She presented at our inpatient clinic 11 months from symptom onset. She had no family history of neuromuscular disease or PDB. Neurologic examination revealed weakness in all four limbs and amyotrophy of both upper limbs. She had increased deep tendon reflexes in both lower limbs and presented bilateral Chaddock and Rossolimo signs, with diminished abdominal reflexes. She also exhibited positive increased palmomental reflexes, without bulbar and respiratory difficulties. Muscle strength of the arms and legs was II and III, respectively. The sensory, autonomic, and extrapyramidal systems were normal. Forced vital capacity was $73 \%$. EMG showed diffuse neurogenic changes in the sternocleidomastoid, trapezius, first interosseous, rectus abdominis and anterior tibia. The waves in the right median, right ulnar nerve and both peroneal nerves were decreased. The CSF showed normal cells and proteins, and the wholebody CT did not show pathological bone formation, resorption, deformity, or fractures. The blood 
chemical analysis showed a slightly lower alkaline phosphatase level (48 u/l, normal 50-135 u/l). Blood tests excluded tumor, thyroid dysfunction and immune system disease, and this patient was diagnosed with clinically definitive ALS. The patient eventually died of respiratory failure 26 months after disease onset.

Patient 5 was a 68 -years-old Han male with a P388S substitution. He noticed bilateral weakness of the upper limbs at age 65 years, bilateral weakness of the legs 10 months later, and bulbar signs after six months. He presented at our outpatient clinic 15 months after symptom onset. He had no family history of neuromuscular disease or PDB. Neurologic examination revealed weakness and amyotrophy in all limbs and mild tongue atrophy and occasional fasciculation. He had increased deep tendon reflexes in both lower limbs, diminished abdominal reflexes, and positive palmomental and sucking reflexes. Forced vital capacity was $85 \%$. The sensory, autonomic, and extrapyramidal systems were normal, and EMG revealed diffuse neurogenic changes in all four limbs and the sternocleidomastoid. The three parts of the MUP were abnormal. The whole-body $\mathrm{X}$-ray did not show pathological bone fractures, and the blood chemical analysis showed a normal alkaline phosphatase level. Other examinations were normal, including blood tests. This patient was diagnosed with probable ALS.

Patient 6 was a 41 -years-old Han male with a P392 L substitution. He had weakness and atrophy in the left arm 36 months prior to visiting the hospital and finally sought treatment after experiencing a decline in swallowing and enunciation 33 months earlier. He was unable to lift his right arm for 30 months and felt weakness of the legs 12 months later. $\mathrm{He}$ had no family history of neuromuscular disease and PDB. Neurologic examination revealed generalized weakness and amyotrophy of the tongue, with bulbar symptoms, diffuse muscle weakness and atrophy of both upper limbs and occasional fasciculation. The deep tendon reflexes were hyperactive in all four limbs with increased palmomental reflexes, and the Hoffman, Babinskii and Chaddock signs were positive. Forced vital capacity was $76 \%$. The sensory, autonomic, and extrapyramidal systems were normal, and EMG showed diffuse neurogenic changes in all four limbs. The whole-body X-ray did not show pathological bone fractures, and the blood chemical analysis showed a normal alkaline phosphatase level. Other examinations were normal, including blood tests. This patient was diagnosed with probable ALS. The symptoms progressed slowly, and he became wheelchair-bound. 